\title{
A Network Pharmacology Based Analysis of The Potential Mechanisms of Compound Danshen Dripping Pill for Treatment of Diabetic Retinopathy
}

\section{Xinyue Zhang}

Chengdu University of Traditional Chinese Medicine https://orcid.org/0000-0003-3762-0740

\section{Lizhen Wang}

Chengdu University of Traditional Chinese Medicine

\section{Xiaoying Huang}

Chengdu University of Traditional Chinese Medicine

Yanlin Zheng ( $\nabla$ zhengyanlin@cdutcm.edu.cn )

Chengdu University of Traditional Chinese Medicine

\section{Research}

Keywords: Danshen, diabetic retinopathy, traditional Chinese medicine, network pharmacology

Posted Date: June 30th, 2020

DOI: https://doi.org/10.21203/rs.3.rs-37961/v1

License: (c) (i) This work is licensed under a Creative Commons Attribution 4.0 International License.

Read Full License 


\section{Abstract}

Background: Diabetic retinopathy (DR) has become a worldwide concern in recent years because of the high prevalence and vision-threat. The limited therapies have been in stark contrast to its high prevalence. On top of that, almost all the treatments, like laser, are applied only at the end stage of this disease. However, with the development of network pharmacology, a traditional Chinese medicine (TCM), Compound Danshen Dripping Pill (CDDP), may bring some new insights into the early intervention of DR.

Methods: The active compounds and potential targets of CDDP and DR-related targets were collected from the TCMSP, UniProt, GeneCards and OMIM databases. And protein-protein interactions (PPI) information was achieved from the STRING database. The gene enrichment analysis of GO and KEGG was carried out by "clusterProfiler" in R software. The collected data was then used to form network maps of compound-target and PPI, or visualized by the software of Cytoscape.

Results: 54 compounds and 50 targets were identified for CDDP against DR. Among the predicted effective compounds, 29 tanshinones from Radix Salviae (Danshen) were identified. And the core targets might be estrogen receptor (ESR1), androgen receptor (AR), caspase-3 (CASP3), Interleukin-6 (IL6) and vascular endothelial growth factor A (VEGFA) in 50 targets. There were 266 significantly correlated biological processes such as steroid hormone response, oxidative stress and apoptosis enriched out. Besides, The 50 targets significantly participate in 97 pathways and mainly enriched in advanced glycation end products (AGE)-receptor for advanced glycation end products (RAGE), fluid shear stress and atherosclerosis, apoptosis and VEGF signal pathway et al.

Conclusions: The mechanisms of CDDP for treating DR may involve multi-compound, multi-target and multi-pathway synergistic effects. It may participate in the regulation of steroid hormone response, oxidative stress, apoptosis and hemodynamics in diabetic retina. Tanshinones and luteolin from Radix Salviae were probably responsible for the effectiveness of CDDP on DR.

\section{Introduction}

Diabetic retinopathy (DR) is common and serious ocular complication of diabetes, with a high blindness rate. The estimated prevalence of DR in China is $34.5 \%$ in people with diabetes mellitus (DM), with $0.99 \%$ for vision-threatening DR. ${ }^{1}$ The rate in rural China is higher than that in urban areas, which is about $43 \%$ in DM including 6.3\% for vision-threatening DR. ${ }^{2}$ At present, the treatments of DR, such as laser, intraocular administration of anti-vascular endothelial growth factor (VEGF) agents and surgical intervention, all aim at reserving impaired vision but not ameliorating. All of these treatments are invasive and carried on the end stage of this disease because of the significant sight-threatening side effects. ${ }^{3}$ Therapies for early retinopathy, except for managing systemic risk factors such as hyperglycemia, hypertension and dyslipidemia et al, is also significant. Modern medical therapies are based on the understanding of pathophysiological mechanisms underlying the development of DR which still need 
plenty of critical efforts. In the meantime, traditional Chinese medicine (TCM), as a different medical system, may provide some clues for preventing or treating DR from the early stage.

The application of TCM in diabetic retinopathy has a long history in China, and some effective TCM treatments for DR have been found in clinical practice. ${ }^{4}$ TCM characterized by multi-compounds might synergistically regulate multi-targets of complex diseases like DR. The developing network biology indicates that complex diseases may involve a cascade of changes that are interactive and should be viewed as a whole. 5,6 This concept is similar to the organic conception of the human body in TCM. Based on the holism, regulating multiple core targets simultaneously could be more effective for complex diseases than single ones. From this point of view, TCM therapies of interest may have some information about the optimum combination of core targets of diseases. However, also limited by the multicompounds and multi-targets of TCM, the researches about the effective compounds or targets of TCM for diseases are relatively difficult and slow. Network pharmacology may provide some insights into the network of biological effects of TCM, and helps to identify core targets of complex diseases.

To understand the mechanism of TCM on DR, we investigated a therapeutic pure TCM drop, Compound Danshen Dripping Pill (CDDP). The effectiveness and safety of CDDP treating or delaying DR have been studied in clinical trials. ${ }^{7,8}$ And it has been recommended for DR treatment. ${ }^{9}$ But the mechanisms underlying the anti-DR effects of CDDP are poorly understood. In this study, with the help of network pharmacology, we analyzed the compounds and targets of CDDP and DR-related targets to explore the potential molecular targets and mechanisms of CDDP for the treatment of DR.

\section{Materials And Methods}

\subsection{Screening active compounds of CDDP}

The recipe composition of CDDP includes the root and rhizome of Radix Salviae (RS, danshen), the root and rhizome of Panax notoginseng (Burk.) F. H. Chen (PN, sanqi) and Borneolum Syntheticum (BS, bingpian). We identified the chemical compounds of each herb of CDDP from Traditional Chinese Medicine Systems Pharmacology Database (TCMSP), ${ }^{10}$ and selected candidate compounds which have oral bioavailability $(\mathrm{OB}) \geq 30 \%$ and drug-likeness $(\mathrm{DL}) \geq 0.18 .{ }^{11}$ Additionally, in combination with relevant literature, ${ }^{12,13}$ the compounds whose biological activity and pharmacological action were reported were also included in the candidate active compounds. Salvianolic acid B and tanshinone I from RS, and L-borneol, D-borneol, isoborneol from BS were included in the follow-up study, although the OB or DL values of the above components are lower than $30 \%$ or 0.18 . Eventually, a main active compounds data set of CDDP was established. The structure, biological activity and other details of the compounds were achieved by the Pubchem database. ${ }^{14}$

\subsection{Identification of potential targets of CDDP}


The primary targets of CDDP was excavated from the TCMSP database. ${ }^{10}$ The target protein name of CDDP was converted into a gene name though the UniProt database. ${ }^{15}$ By inputting the target protein name and defining the species as "Homo sapiens", the non-human targets were eliminated.

\section{3. collection of DR-related Targets}

The differential expressed genes of DR were obtained from the GeneCards database ${ }^{16}$ and the OMIM database ${ }^{17}$ with the keyword of "Diabetic Retinopathy." Genes with a P-value $<0.05$ were considered to be of significant differential expression in DR.

\subsection{Network Construction and Merge}

Venn analysis was carried out to achieve the common target gene set of CDDP and DR through the online platform of Bioinformatics and Evolutionary Genomics. ${ }^{18}$ The network of "CDDP-compound-target-DR " was constructed and visualized with Cytoscape 3.7.1 software and analyzed with Network Analyzer.

PPI data was achieved from the STRING database. Data that the minimum required interaction score > 0.4 was chosen. The network of protein-protein interaction visualized with Cytoscape software and core targets were selected according to the degree value of the nodes.

\subsection{Bioinformatic analysis}

Bioconductor package "org.Hs.eg.db" was used to convert the target into entrezID in R software. According to the transformed entrezID, "clusterProfiler" was used to perform Gene Ontology (GO) and Kyoto Encyclopedia of Genes and Genomes (KEGG) functional enrichment analysis of the target genes with significant changes of $P<0.05$ and $Q<0.05$. The top 20 significant categories of $G O$ and KEGG analysis were carried out in the form of a bar chart.

\section{Results}

\subsection{Compound-target analysis of CDDP}

The network of compound-target was carried out as Figure 1. Eighty-one compounds of CDDP were identified as the candidate compounds from the TCMSP database. The total 81 compounds were input into the UniProt database to identify the corresponding targets. Seventeen compounds were deleted because no relevant targets have been recorded yet in the database. After deduplication, a total of 64 potential active ingredients were finally obtained (Figure 1, the outer circle). RS, PN, and BS had 55 (Figure 1, red dot), 7 (Figure 1, green dot), 2 (Figure 1, yellow dot) active compounds, respectively. These compounds had a median degree value (edges) of 9.22, indicating a multi-targeting effect of CDDP, among which quercetin, luteolin and tanshinone IIA had the highest degree value of 78, 29 and 22. Sixtyfour compounds were related to 109 targets (Figure 1, the inner grid). The total targets of CDDP in our study were shown highly related to nuclear hormone receptor superfamily such as nuclear receptor 
coactivator (NCOA), prostaglandin G/H synthase 1 (PTGS1), alpha-1A adrenergic receptor (ADRA1A), estrogen receptor 1 (ESR1), androgen receptor (AR) and cholinergic signal regulators including muscarinic acetylcholine receptor M1-5 (CHRM1-5) and acetylcholinesterase (ACHE), according to the degree value of nodes.

\subsection{The candidate targets of CDDP against DR}

The 109 CDDP targets were merged with 2702 DR-related target genes. After the intersection of the two, 50 candidate targets of CDDP against DR were identified (Figure 2a), with 27 targets of RS (Figure 2a), 43 targets of PN (Figure 2a), and 1 target of BS (Figure 2a). The 50 targets were related to 54 active compounds of CDDP (Table 1). Quercetin and luteolin, had the highest degree value of 39 and 17. And 29 tanshinones (Table 1superscript) were identified. These compounds may be the representative compounds of CDDP against DR. Eventually, the interaction of 54 potential effective compounds and 50 candidate targets was formed the network of CDDP-compound-target-DR (Figure 2b). ACHE, ESR1 and AR were maximally targeted with 26,20 and 20 edges, respectively.

\subsection{PPI networks analysis}

In order to reveal the mechanisms of action underlying CDDP's effects on DR, the PPI network analysis was applied to show the protein interaction network of the candidate targets of CDDP against DR (Figure 3). Interleukin-6 (IL6), epidermal growth factor receptor (EGFR), CASP3 and VEGFA with the degree of 38, 37, 37 and 36, respectively, might be the core targets in the network (Figure 3a). To better understand the role of CDDP in the PPI network, we merged the PPI network and the CDDP-compound-target-DR network (Figure $\mathbf{2 b}$ ) as Figure $\mathbf{3 b}$. After the intersection, new order of the nodes was formed based on degree value. The top 5 targets were ESR1, AR, CASP3, IL 6 and VEGFA with 50, 46, 42, 40 and 39 edges, respectively.

\subsection{GO and KEGG enrichment analysis}

To analysis what biological processes CDDP might involve in when applied to DR, GO analysis was carried out. $266 \mathrm{GO}$ terms were significantly enriched (P-adjust $<0.05$ ) based on the biological process (Supplementary Table 1). The top 20 terms were shown in Figure 4. The highly enriched GO terms included steroid hormone response, oxidative stress, apoptosis et al.

The pathways that were significantly influenced by CDDP for the treatment of DR were identified by KEGG pathway analysis. 97 significantly enriched pathways were identified (Supplementary Table 2), and the top 20 pathways were shown in Figure 5. The results showed that the common targets were mainly concentrated in the advanced glycation end-products and receptor for advanced glycation end-products (AGE-RAGE) signaling pathway, fluid shear stress and atherosclerosis, apoptosis, tumor necrosis factor (TNF), hypoxia-inducible factor-1 (HIF-1), phosphoinositide 3-kinase (PI3K)/protein kinase B (Akt) and 
VEGF signaling pathway, which regulate many biological processes like oxidative stress, blood pressure, hemodynamic change, apoptosis, inflammation, retinal ischemia, proliferation and differentiation. ${ }^{19-26}$

\section{Discussion}

In TCM theory, RS is supposed to be the most responsible herb for CDDP's effects. According to our putative active compounds of CDDP for DR, RS has 48 among a total of 54 active compounds with 27 targets. 45 of 48 active compounds are specific in the herb, indicating that RS may play a vital role in the formula consistent with TCM theory. RS have shown some anti-diabetic properties by regulating multiple pathways and biological processes related to the development of diabetes. ${ }^{27}$ Thus anti-diabetic compounds are expected to be identified from it. In our study, Tanshinones were identified as the major effective compounds of RS demonstrating multiple bioactivities. It is suggested that tanshinones could be therapeutic agents for diabetes as insulin sensitizer and AMPK pathway activators. ${ }^{28-30}$ Early application of tanshinones may prevent the progress of DR. Besides, many tanshinones have shown antiinflammatory effect, ${ }^{31}$ and total tanshinones exhibit a stronger effect than a single one. ${ }^{32}$ Since inflammation is supposed to play a vital role in DR, the application of tanshinones could also be beneficial for DR in this respect. Among the tanshinones, tanshinone IIA stood out with the highest node degree. It has been suggested exerting anti-angiogenesis, anti-oxidative stress, anti-inflammatory effects in the retina through suppressing VEGF, AGE, NF-KB pathway. ${ }^{33-35}$ There were still some non-specific compounds of CDDP at the top of the list (Table 1), which could not be ignored. Polyphenols like quercetin and luteolin have been suggested to be therapeutic for DR. ${ }^{36}$ Luteolin from RS is believed to have effects on activating anti-inflammatory, antioxidant responses, neuroprotective effects and regulating relaxation, lipid profile via acting on multiple signaling pathways. ${ }^{37-42}$ Taken together, tanshinones and luteolin may be responsible for the effectiveness of CDDP against DR.

For PN, although 5 compounds have 43 targets, most of them like quercetin could be found in plenty of Chinese herbs, and PN is less dosage in the formula, the compounds identified were not suggested as the representative compounds for CDDP. The situation of BS is almost the same as PN. But their compatibility with RS is necessary according to TCM theory. Pharmacokinetics showed that the bioavailability of PN extract significantly increased when co-administered with BS through oral administration in rats. ${ }^{43}$ It suggests that BS might work as a potential absorption enhancer, and an upper ushering to brain drug. ${ }^{44}$ Although there is no significant difference in pharmacokinetic parameters between single RS or PN extract and combination treatment through oral administration, ${ }^{43}$ the combination treatment enhanced RS compounds in the brain and cerebrospinal fluid while decreasing the distribution of PN compounds via inner ear administration in guinea pigs. ${ }^{45}$ These researches indicate that herb-herb interactions may improve pharmacokinetic behaviors of CDDP's active compounds.

Our results indicated that the predominant targets of CDDP against DR were ESR1, AR, CASP3, IL6 and VEGFA. ESR and AR are steroid hormone receptor. ESR signaling has shown its importance in ischemiainduced angiogenesis ${ }^{46,47}$ and neural injury gradually ${ }^{48-50}$. But the effects of sex hormones in DR are not 
fully understood and quite controversial at present. ${ }^{51}$ Estrogen could be neuroprotective $\mathrm{e}^{52,53}$ or useless ${ }^{54}$ or harmful ${ }^{55}$. Its neuroprotective effect may be related to anti-apoptosis and anti-autophagy effects, while androgen is more pro-apoptotic ${ }^{56}$. From our results, CDDP could have a potential influence on both ESR and AR signaling. Furthermore, CDDP showed a potential influence on CASP-3, a downstream executioner of apoptosis in DR, ${ }^{57-60}$ in our study and its inhibitory effect on CASP-3 expression has been reported. ${ }^{61}$ Combining with the GO enrichment analysis, CDDP was strongly indicated involved in the regulation of steroid hormone response and apoptosis in DR.

Chronic inflammation has been suggested by growing evidence playing a prominent role in the pathogenesis of DR. ${ }^{62-64} \mathrm{IL}-6$, one of the inflammatory mediators, is found elevated in diabetic retinopathy. ${ }^{65-67}$ IL-6 is a pleiotropic cytokine, and its effects could be quite different through activating different pathways. Binding to membrane-bound IL- 6 receptor and gp130, IL-6 activates classical IL-6 signaling pathway, which is supposed to be regenerative and protective; while binding to the soluble IL- 6 receptor, IL-6 activates IL-6 trans-signaling pathway which is chronic pro-inflammatory and resulting in endothelial cell dysfunction and vascular leakage. ${ }^{68-70}$ both pathways could be activated in the retina under high glucose. ${ }^{71,72}$ Meanwhile, IL-6 is highly correlated with the aqueous level of VEGF in DR. ${ }^{73}$ The $^{7}$ correlation between VEGF and the pathogenesis of DR is uncontroversial, especially for proliferative DR. ${ }^{21,74,75}$ Downstream events of VEGF include survival, proliferation, and permeability. ${ }^{76}$ It is supposed to improve the survival of retinal cells in the first place. But over time, it disrupts the outer blood-retinal barriers (BRB) and acts as a pathologic angiogenic stimulus. ${ }^{77,78}$ These studies indicate the feasibility of CDDP against DR by targeting these predicted targets and their biological processes. Our results showed that CDDP might regulate multiple targets of DR, including inflammatory cytokines and growth factors et al.

At present, several biological processes and pathways have been proposed to participate in the development of DR including oxidative stress, AGE, protein kinase $C$ (PKC) activation, inflammation, VEGF, sorbitol, erythropoietin, the renin-angiotensin system (RAS), peroxisome proliferator-activated receptor a (PPAR-a). ${ }^{79,80}$ These processes or pathways could be the potential therapeutic targets for DR. As our results indicated, the mechanisms underlying the effect of CDDP for DR might involve in multiple pathways. A total of 97 KEGG pathways were enriched out including the AGE-RAGE and VEGF pathway, fluid shear stress and atherosclerosis, apoptosis et al. AGE-RAGE and VEGF pathways play critical roles in DR and has been discussed a lot. ${ }^{19-21,81-87}$ Except for the biochemical changes, physiological changes also play a role in the process of DR. Hemodynamic dysfunction has been found in DR and may precede clinical DR. ${ }^{88-92}$ Hemodynamic parameters, wall shear rate and wall shear stress are suggested reduced in the early stage of DR, as well as retinal blood velocity and flow, which could be markers of microvasculopathy ${ }^{88,93,94}$ Atherosclerosis has also been considered associated with the severity of DR, manifesting increased macrovascular artery intima-media wall thickness and accompanied by wider retinal vessel diameter in DR. ${ }^{95-97}$ These studies emphasize the vital role of hemodynamic changes in the early stage of DR. CDDP in TCM theory is characterized by improving blood circulation 
(Pharmacopoeia of the People's Republic of China). The early application of CDDP in NPDR patients can significantly shorten the retinal circulation time and reduce capillary hemangioma, hard exudates, retinal hemorrhaging. ${ }^{8}$ And CDDP may also have an anti-atherosclerosis property. ${ }^{98}$ The potential regulation on hemodynamics through fluid shear stress and atherosclerosis pathway may make CDDP a suitable medicine for an early intervention of DR. Besides, fluid shear stress is related to the release of

vasodilatory factors nitric oxide (NO). ${ }^{99-101}$ As we all know, blood pressure has been suggested as one of the risk factors of DR. ${ }^{102}$ The regulation of fluid shear stress and atherosclerosis pathway by CDDP may affect blood pressure control. And anti-hypertension of RS has been reported by some researches. ${ }^{103-105}$ In summary, CDDP may be beneficial in the early stage of DR by acting on AGE-RAGE, VEGF, fluid shear stress and atherosclerosis, and apoptosis pathway.

Except for vascular dysfunction, retinal neurodegeneration has been recognized in DR with growing evidence. New insights suggest that vascular dysfunction and neural degeneration may be parallel in this disease. Thus physiological changes of DR should be viewed as changes of retinal neurovascular unit. ${ }^{79,106}$ The unit includes the interaction of vascular cells, retinal glial cells and neural cells. Because of the interaction, elevated pro-inflammatory or pro-angiogenesis cytokines in DR may contribute to both the blood-retina barrier (BRB) disruption and synaptic or neuronal degeneration. ${ }^{79}$ Cholinergic signaling may be blunted in diabetic retina, which may associate with synaptic degeneration, resulting in endotheliumdependent vasodilatation dysfunction and ischemia. ${ }^{107,108}$ And CHRM has been suggested to play an important role in retinal neuron survival ${ }^{109,110}$ and insulin release stimulation. ${ }^{111-113}$ It is rational to hypothesize that active cholinergic signaling could be protective in DR. In the compound-target network of CDDP, it showed that CDDP might have a potential regulation on cholinergic signaling through CHRM15 and ACHE (Fig. 1). Although it is not clear what exact role of cholinergic signaling plays in DR, therapies targeting cholinergic signaling could be worth trying considering the neuroprotective effects of its agonists towards other neural degenerative diseases like Alzheimer's and Parkinson's diseases. ${ }^{114}$

\section{Conclusions}

The relatively scarce data of CDDP in the public TCM database and the incomplete interaction of protein interactome may constrain our results. Nevertheless, we identified some very likely candidates to explain the mechanisms behind the effect of CDDP against DR. ESR1, AR, CASP3, IL6 and VEGFA may be the center targets in the action network of CDDP against DR involved in steroid hormone response, oxidative stress and apoptosis processes. Regulation of steroid hormone response may be the primary mechanism of CDDP for DR. But the exact role of steroid hormone in DR needs more investigations. Except for the well-known DR-related AGE-RAGE, apoptosis and VEGF pathway, CDDP has been indicated to participate in the regulation of fluid shear stress and atherosclerosis pathway. It seems that CDDP could have a potential effect on hemodynamics consistent with TCM theory. These results indicated that the early application of CDDP in DR might be beneficial from hemodynamics, oxidative stress and apoptosis improvement. And the therapeutic compounds may be tanshinones and luteolin, since the regulation of most of the targets. In summary, this study showed that the underlying mechanism of CDDP treating DR 
might involve multi-compound, multi-target and multi-pathway synergistic effects. Those data may bring new insights for further investigations and new strategies for the prevention or treatment of DR from an early stage.

\section{Abbreviations}




\begin{tabular}{|c|c|}
\hline DR & Diabetic retinopathy \\
\hline CDDP & Compound Danshen Dripping Pill \\
\hline TCM & Traditional Chinese medicine \\
\hline DM & Diabetes mellitus \\
\hline VEGF & Vascular endothelial growth factor \\
\hline RS & Radix salviae \\
\hline PN & Panax notoginseng \\
\hline BS & Borneolum syntheticum \\
\hline TCMSP & Traditional Chinese Medicine Systems Pharmacology \\
\hline NCOA & Nuclear receptor coactivator \\
\hline PTGS & Prostaglandin G/H synthase \\
\hline ADRA1A & Alpha- $1 \mathrm{~A}$ adrenergic receptor \\
\hline ESR & Estrogen receptor \\
\hline AR & Androgen receptor \\
\hline CHRM & Muscarinic acetylcholine receptor $\mathrm{M}$ \\
\hline $\mathrm{ACHE}$ & Acetylcholinesterase \\
\hline PPI & Protein-protein interactions \\
\hline IL6 & Interleukin-6 \\
\hline EGFR & Epidermal growth factor receptor \\
\hline CASP3 & Caspase-3 \\
\hline GO & Gene Ontology \\
\hline KEGG & Kyoto Encyclopedia of Genes and Genomes \\
\hline AGE & Advanced glycation end-products \\
\hline RAGE & Receptor for advanced glycation end-products \\
\hline TNF & Tumor Necrosis Factor \\
\hline HIF & Hypoxia-inducible factor \\
\hline BRB & Blood-retina barrier \\
\hline PKC & Protein kinase $\mathrm{C}$ \\
\hline RAS & renin-angiotensin system \\
\hline PPAR-a & Peroxisome proliferator-activated receptor a \\
\hline
\end{tabular}


AMPK Adenosine 5'-monophosphate (AMP)-activated protein kinase

NF-kB Nuclear factor kappa-B

\section{Declarations}

Author Contributions: X.Z. and L.W. contributed to this article equally. Conceptualization, X.Z. and L.W.; methodology, X.Z. and L.W.; writing-original draft preparation, X.Z. and L.W.; writing-review and editing, X.H.; supervision, Y.Z. All authors have read and agreed to the published version of the manuscript.

Data Availability Statement: The datasets used during the current study are available from the corresponding author on reasonable request.

Funding: This research received no external fund.

Conflicts of Interest: The authors declare that there are no conflicts of interest.

Consent for publication: Written informed consent for publication was obtained from all participants.

\section{References}

1. Song P, Yu J, Chan KY, et al. Prevalence, risk factors and burden of diabetic retinopathy in China: a systematic review and meta-analysis. J Glob Health. 2018;8:010803. https://doi.org/10.7189/jogh.08.010803.

2. Wang FH, Liang YB, Zhang F, et al. Prevalence of Diabetic Retinopathy in Rural China: The Handan Eye Study. Ophthalmology. 2009;116:461-467. https://doi.org/10.1016/j.ophtha.2008.10.003.

3. Simó R, Hernández C. Advances in the Medical Treatment of Diabetic Retinopathy. Diabetes Care. 2009;32:1556-1562. https://doi.org/10.2337/dc09-0565.

4. Xu ZH, Gao YY, Zhang HT, et al. Progress in Experimental and Clinical Research of the Diabetic Retinopathy Treatment Using Traditional Chinese Medicine. Am J Chin Med. 2018;1-27. https://doi.org/10.1142/S0192415X1850074X.

5. Barabási AL, Oltvai ZN. Network biology: understanding the cell's functional organization. Nat Rev Genet. 2004;5:101-113. https://doi.org/1038/nrg1272.

6. Hopkins AL. Network pharmacology. Nat Biotechnol. 2007;25:1110-1111. https://doi.org/10.1038/nbt1007-1110.

7. Lian F, Wu L, Tian J, et al. The effectiveness and safety of a danshen-containing Chinese herbal medicine for diabetic retinopathy: a randomized, double-blind, placebo-controlled multicenter clinical trial. J Ethnopharmacol. 2015;164:71-77. https://doi.org/10.1016/j.jep.2015.01.048.

8. Huang W, Bao Q, Jin, Lian F. Compound Danshen Dripping Pill for Treating Nonproliferative Diabetic Retinopathy: A Meta-Analysis of 13 Randomized Controlled Trials. Evid Based Complement Alternat Med. 2017;4848076. https://doi.org/10.1155/2017/4848076. 
9. Expert Panel from Chinese Geriatrics Society on the Clinical Use of Compound Danshen Dripping Pills. Chin Med J (Engl). 2017;130:972-978. https://doi.org/10.4103/0366-6999.204106.

10. The Traditional Chinese Medicine Systems Pharmacology Database. http://lsp.nwu.edu.cn/tcmsp.php.

11. Li J, Zhao P, Li Y, et al. Systems pharmacology-based dissection of mechanisms of Chinese medicinal formula Bufei Yishen as an effective treatment for chronic obstructive pulmonary disease. Sci Rep. 2015;5:15290. https://doi.org/10.1038/srep15290.

12. Ying Z, Lingliang G, Lin B, Xuechun S, et al. Research Progress on Borneolum. Chinese Journal of Tropical Agriculture. 2019;39:97-104. https://doi.org/10.12008/j.issn.1009-2196.2019.03.017.

13. Qi Z, Huiting F, Ying Z, et al. Progress of chemical composition analysis of Salviae Miltiorrhizae and its antitumor pharmacological effects. Chinese Archives of Traditional Chinese Medicine. Available online: http://kns.cnki.net/kcms/detail/21.1546.r.20190815.1015.042.html.

14. The Pubchem database. https://pubchem.ncbi.nlm.nih.gov/.

15. The UniProt database. https://www.uniprot.org/.

16. The GeneCards database. https://www.genecards.org/.

17. The OMIM database. https://www.omim.org/.

18. The Bioinformatics and Evolutionary Genomics. http://bioinformatics.psb.ugent.be/webtools/Venn/.

19. Stitt AW. AGEs and diabetic retinopathy. Invest Ophthalmol Vis Sci. 2010;51:4867-4874. https://doi.org/10.1167/iovs.10-5881.

20. Barile GR, Pachydaki SI, Tari SR, et al. The RAGE axis in early diabetic retinopathy. Invest Ophthalmol Vis Sci. 2005;46:2916-24. https://doi.org/10.1167/iovs.04-1409.

21. Wirostko B, Wong TY, Simó R. Vascular endothelial growth factor and diabetic complications. Prog Retin Eye Res. 2008;27:608-621. https://doi.org/10.1016/j.preteyeres.2008.09.002.

22. Jo DH, Yun JH, Cho CS, et al. Interaction between microglia and retinal pigment epithelial cells determines the integrity of outer blood-retinal barrier in diabetic retinopathy. Glia. 2019;67:321-331. https://doi.org/10.1002/glia.23542.

23. Capitão M, Soares R. Angiogenesis and Inflammation Crosstalk in Diabetic Retinopathy. J Cell Biochem. 2016;117:2443-2453. https://doi.org/10.1002/jcb.25575.

24. Gao X, Li Y, Wang H, et al. Inhibition of HIF-1 a decreases expression of pro-inflammatory IL-6 and TNF-a in diabetic retinopathy. Acta Ophthalmol. 2017;95:e746-e750. https://doi.org/10.1111/aos.13096.

25. Jere SW, Houreld NN, Abrahamse H. Role of the PI3K/AKT (mTOR and GSK3 3 ) signalling pathway and photobiomodulation in diabetic wound healing. Cytokine Growth Factor Rev. 2019;50:52-59. https://doi.org/11016/j.cytogfr.2019.03.001.

26. Elsherbiny NM, Abdel-Mottaleb Y, Elkazaz AY, et al. Carbamazepine Alleviates Retinal and Optic Nerve Neural Degeneration in Diabetic Mice via Nerve Growth Factor-Induced PI3K/Akt/mTOR Activation. Front Neurosci. 2019;13:1089. https://doi.org/10.3389/fnins.2019.01089. 
27. Jia Q, Zhu R, Tian Y, et al. Salvia miltiorrhiza in diabetes: A review of its pharmacology, phytochemistry, and safety. Phytomedicine. 2019;58:152871. https://doi.org/10.1016/j.phymed.2019.152871.

28. Kim DH, Paudel P, Yu T, et al. Characterization of the inhibitory activity of natural tanshinones from Salvia miltiorrhiza roots on protein tyrosine phosphatase 1B. Chem Biol Interact. 2017;278:65-73. https://doi.org/10.1016/j.cbi.2017.10.013.

29. Jung SH, Seol HJ, Jeon SJ, et al. Insulin-sensitizing activities of tanshinones, diterpene compounds of the root of Salvia miltiorrhiza Bunge. Phytomedicine. 2009;16:327-335. https://doi.org/10.1016/j.phymed.2008.12.017.

30. Kim EJ, Jung SN, Son KH, et al. Antidiabetes and antiobesity effect of cryptotanshinone via activation of AMP-activated protein kinase. Mol Pharmacol. 2007;72:62-72. https://doi.org/10.1124/mol.107.034447.

31. Ma S, Zhang D, Lou H, et al. Evaluation of the anti-inflammatory activities of tanshinones isolated from Salvia miltiorrhiza var. alba roots in THP-1 macrophages. J Ethnopharmacol. 2016;188:193199. https://doi.org/10.1016/j.jep.2016.05.018.

32. Gao H, Huang L, Ding F, et al. Simultaneous purification of dihydrotanshinone, tanshinone I, cryptotanshinone, and tanshinone IIA from Salvia miltiorrhiza and their anti-inflammatory activities investigation. Sci Rep. 2018;8:8460. https://doi.org/10.1038/s41598-018-26828-0.

33. Guo ZL, Li Y, Liu XW, et al. Sodium Tanshinone IIA Silate Alleviates High Glucose Induced Barrier Impairment of Human Retinal Pigment Epithelium through the Reduction of NF-KB Activation via the AMPK/p300 Pathway. Curr Eye Res. 2020;45:177-183. https://doi.org/10.1080/02713683.2019.1668419.

34. Qian S, Qian Y, Huo D, et al. Tanshinone lla protects retinal endothelial cells against mitochondrial fission induced by methylglyoxal through glyoxalase 1. Eur J Pharmacol. 2019;857:72419. https://doi.org/10.1016/j.ejphar.2019.172419.

35. Fan K, Li S, Liu G, et al. Tanshinone IIA inhibits high glucose-induced proliferation, migration and vascularization of human retinal endothelial cells. Mol Med Rep. 2017;16:9023-9028. https://doi.org/10.3892/mmr.2017.7743.

36. Rodríguez ML, Pérez S, Mena-Mollá S, et al. Oxidative Stress and Microvascular Alterations in Diabetic Retinopathy: Future Therapies. Oxid Med Cell Longev. 2019;4940825. https://doi.org/10.1155/2019.

37. Yan J, Zhang G, Pan J, et al. a-Glucosidase inhibition by luteolin: kinetics, interaction and molecular docking. Int J Biol Macromol. 2014;64:213-23. https://doi.org/10.1016/j.ijbiomac.2013.12.007.

38. Li L, Luo W, Qian Y, et al. Luteolin protects against diabetic cardiomyopathy by inhibiting NF-KBmediated inflammation and activating the Nrf2-mediated antioxidant responses. Phytomedicine. 2019;59:152774. https://doi.org/10.1016/j.phymed.2018.11.034.

39. Ambasta RK, Gupta R, Kumar D, et al. Can luteolin be a therapeutic molecule for both colon cancer and diabetes. Brief Funct Genomics. 2018;18:230-239. https://doi.org/10.1093/bfgp/ely036. 
40. Nabavi SF, Braidy N, Gortzi O, et al. Luteolin as an anti-inflammatory and neuroprotective agent: A brief review. Brain Res Bull. 2015;119:1-11. https://doi.org/10.1016/j.brainresbull.2015.09.002.

41. Kwon EY, Jung UJ, Park T, et al. Luteolin attenuates hepatic steatosis and insulin resistance through the interplay between the liver and adipose tissue in mice with diet-induced obesity. Diabetes. 2015;64:1658-1669. https://doi.org/10.2337/db14-0631.

42. El-Bassossy HM, Abo-Warda SM, Fahmy A. Chrysin and luteolin alleviate vascular complications associated with insulin resistance mainly through PPAR-y activation. Am J Chin Med. 2014;42:11531167. https://doi.org/10.1142/S0192415X14500724.

43. Yang S, Zhang K, Lin X, et al. Pharmacokinetic comparisons of single herb extract of Fufang Danshen preparation with different combinations of its constituent herbs in rats. J Pharm Biomed Anal. 2012;67-68:77-85. https://doi.org/10.1016/j.jpba.2012.03.058.

44. Chen ZX, Xu QQ, Shan CS, et al. Borneol for Regulating the Permeability of the Blood-Brain Barrier in Experimental Ischemic Stroke: Preclinical Evidence and Possible Mechanism. Oxid Med Cell Longev. 2019;2936737. https://doi.org/10.1155/2019/2936737.

45. Long W, Zhang SC, Wen L, et al. In vivo distribution and pharmacokinetics of multiple active components from Danshen and Sanqi and their combination via inner ear administration. $J$ Ethnopharmacol. 2014;156:199-208. https://doi.org/10.1016/j.jep.2014.08.041.

46. Giddabasappa A, Eswaraka JR, Barrett CM, et al. $\beta$-LGND2, an ER $\beta$ selective agonist, inhibits pathologic retinal neovascularization. Invest Ophthalmol Vis Sci. 2012;53:5066-5075. https://doi.org/10.1167/iovs.12-9627.

47. Wu Y, Zhang Q, Zhang R. Kaempferol targets estrogen-related receptor $a$ and suppresses the angiogenesis of human retinal endothelial cells under high glucose conditions. Exp Ther Med. 2017;14:5576-5582. https://doi.org/10.3892/etm.2017.5261.

48. Raval AP, Bramlett $H$, Perez-Pinzon MA. Estrogen preconditioning protects the hippocampal CA1 against ischemia. Neuroscience. 2006;141:1721-1730. https://doi.org/10.1016/j.neuroscience.2006.05.016.

49. Liu H, Yang J, Yang W, et al. Focus on Notoginsenoside R1 in Metabolism and Prevention Against Human Diseases. Drug Des Devel Ther. 2020;14:551-565. https://doi.org/10.2147/DDDT.S240511.

50. Wang Y, Tu L, Li Y, et al. Notoginsenoside R1 Protects against Neonatal Cerebral Hypoxic-lschemic Injury through Estrogen Receptor-Dependent Activation of Endoplasmic Reticulum Stress Pathways. J Pharmacol Exp Ther. 2016;357:591-605. https://doi.org/10.1124/jpet.115.230359.

51. Maric-Bilkan C. Sex differences in micro- and macro-vascular complications of diabetes mellitus. Clin Sci (Lond). 2017;131:833-846. https://doi.org/10.1042/CS20160998.

52. Hao M, Li Y, Lin W, et al. Estrogen prevents high-glucose-induced damage of retinal ganglion cells via mitochondrial pathway. Graefes Arch Clin Exp Ophthalmol. 2015;253:83-90. https://doi.org/10.1007/s00417-014-2771-7.

53. Nuzzi R, Scalabrin S, Becco A, et al. Gonadal Hormones and Retinal Disorders: A Review. Front Endocrinol (Lausanne). 2018;9:66. https://doi/org/10.3389/fendo.2018.00066. 
54. Klein BE, Klein R, Moss SE. Exogenous estrogen exposures and changes in diabetic retinopathy. The Wisconsin Epidemiologic Study of Diabetic Retinopathy. Diabetes Care. 1999;22:1984-1987. https://doi.org/10.2337/diacare.22.12.1984.

55. Espinosa-Heidmann DG, Marin-Castano ME, Pereira-Simon S, et al. Gender and estrogen supplementation increases severity of experimental choroidal neovascularization. Exp Eye Res. 2005;80:413-423. https://doi/org/10.1016/j.exer.2004.10.008.

56. Wnuk A, Kajta M. Steroid and Xenobiotic Receptor Signalling in Apoptosis and Autophagy of the Nervous System. Int J Mol Sci. 2017;18: https://doi.org/10.3390/ijms18112394.

57. Wolf BB, Schuler M, Echeverri F, et al. Caspase-3 is the primary activator of apoptotic DNA fragmentation via DNA fragmentation factor-45/inhibitor of caspase-activated DNase inactivation. $J$ Biol Chem. 1999;274:30651-30656. https://doi.org/10.1074/jbc.274.43.30651.

58. Mohr S, Xi X, Tang J, et al. Caspase activation in retinas of diabetic and galactosemic mice and diabetic patients. Diabetes. 2002;51:1172-1179. https://doi.org/10.2337/diabetes.51.4.1172.

59. Kowluru RA, Koppolu P. Diabetes-induced activation of caspase-3 in retina: effect of antioxidant therapy. Free Radic Res. 2002;36:993-999. https://doi.org/10.1080/1071576021000006572.

60. Tian M, Liu S, Liu L, et al. Correlations of the severity of diabetic retinopathy with EPO, Caspase-3 expression and oxidative stress. Eur Rev Med Pharmacol Sci. 2019;23:9707-9713. https://doi.org/10.26355/eurrev_201911_19532.

61. Zhang Q, Xiao X, Zheng J, et al. Compound Danshen Dripping Pill Inhibits Retina Cell Apoptosis in Diabetic Rats. Front Physiol. 2018;9:501. https://doi.org/10.3389/fphys.2018.01501.

62. Mesquida $M$, Drawnel F, Fauser $S$. The role of inflammation in diabetic eye disease. Semin Immunopathol. 2019;41:427-445. https://doi.org/10.1007/s00281-019-00750-7.

63. Rübsam A, Parikh S, Fort PE. Role of Inflammation in Diabetic Retinopathy. Int J Mol Sci. 2018;19:942. https://doi.org/10.3390/ijms19040942.

64. Tang J, Kern TS. Inflammation in diabetic retinopathy. Prog Retin Eye Res. 2011;30:343-358. https://doi.org/10.1016/j.preteyeres.2011.05.002.

65. Myśliwiec M, Balcerska A, Zorena $K$, et al. The role of vascular endothelial growth factor, tumor necrosis factor alpha and interleukin- 6 in pathogenesis of diabetic retinopathy. Diabetes Res Clin Pract. 2008;79:141-146. https://doi.org/10.1016/j.diabres.2007.07.011.

66. Arjamaa O, Pöllönen M, Kinnunen K, et al. Increased IL-6 Levels Are Not Related to NF-KB or HIF-1a Transcription Factors Activity in the Vitreous of Proliferative Diabetic Retinopathy. J Diabetes Complications. 2011;25:393-397. https://doi.org/10.1016/j.jdiacomp.2011.06.002.

67. Yao Y, Li R, Du J, et al. Interleukin-6 and Diabetic Retinopathy: A Systematic Review and MetaAnalysis. Curr Eye Res. 2019;44:564-574. https://doi.org/10.1080/02713683.2019.1570274.

68. Rose-John S. Interleukin-6 Family Cytokines. Cold Spring Harb Perspect Biol. 2018;10:a028415. https://doi.org/10.1101/cshperspect.a028415. 
69. Jones SA, Jenkins BJ. Recent insights into targeting the IL-6 cytokine family in inflammatory diseases and cancer. Nat Rev Immunol. 2018;18:773-789. https://doi.org/10.1038/s41577-018-00667 .

70. Valle ML, Dworshak J, Sharma A, et al. Inhibition of interleukin- 6 trans-signaling prevents inflammation and endothelial barrier disruption in retinal endothelial cells. Exp Eye Res. 2019;178:2736. https://doi.org/10.1016/j.exer.2018.09.009.

71. Chen H, Zhang X, Liao N, et al. Increased levels of IL-6, sIL-6R, and sgp130 in the aqueous humor and serum of patients with diabetic retinopathy. Mol Vis. 2016;22:1005-1014.

http://www.ncbi.nlm.nih.gov/pmc/articles/pmc4978206/.

72. Coughlin BA, Trombley BT, Mohr S. Interleukin-6 (IL-6) mediates protection against glucose toxicity in human Müller cells via activation of VEGF-A signaling. Biochem Biophys Res Commun. 2019;517:227-232. https://doi.org/10.1016/j.bbrc.2019.07.044.1.

73. Funatsu H, Yamashita H, Shimizu E, et al. Relationship between vascular endothelial growth factor and interleukin-6 in diabetic retinopathy. Retina. 2001;21:469-477. https://doi.org/10.1097/00006982-200110000-00009.

74. Cheung N, Wong IY, Wong TY. Ocular anti-VEGF therapy for diabetic retinopathy: overview of clinical efficacy and evolving applications. Diabetes Care. 2014;37:900-905. https://doi.org/10.2337/dc131990.

75. Stitt AW, Curtis TM, Chen M, et al. The progress in understanding and treatment of diabetic retinopathy. Prog Retin Eye Res. 2016;51:56-86. https://doi.org/10.1016/j.preteyeres.2015.08.001.

76. Simó R, Sundstrom JM, Antonetti DA. Ocular Anti-VEGF therapy for diabetic retinopathy: the role of VEGF in the pathogenesis of diabetic retinopathy. Diabetes Care. 2014;37:893-899. https://doi.org/10.2337/dc13-2002.

77. Jo DH, Yun JH, Cho CS, et al. Interaction between microglia and retinal pigment epithelial cells determines the integrity of outer blood-retinal barrier in diabetic retinopathy. Glia. 2019;67:321-331. https://doi.org/10.1002/glia.23542.

78. Gariano RF, Gardner TW. Retinal angiogenesis in development and disease. Nature. 2005;438:960966. https://doi.org/10.1038/nature04482.

79. Antonetti DA, Klein R, Gardner TW. Diabetic retinopathy. N Engl J Med. 2012;366:1227-1239. https://doi.org/10.1056/NEJMra1005073.

80. Cheung N, Mitchell P, Wong TY. Diabetic retinopathy. Lancet. 2010;376:124-136. https://doi.org/10.1016/S0140-6736(09)62124-3.

81. Wang Y, Vom Hagen F, Pfister F, et al. Receptor for advanced glycation end product expression in experimental diabetic retinopathy. Ann N Y Acad Sci. 2008;1126:42-45. https://doi.org/10.1196/annals.1433.063.

82. Wautier JL, Schmidt AM. Protein glycation: a firm link to endothelial cell dysfunction. Circ Res. 2004;95:233-238. https://doi.org/10.1161/01.RES.0000137876.28454.64. 
83. Zong H, Ward M, Madden A, et al. Hyperglycaemia-induced pro-inflammatory responses by retinal Müller glia are regulated by the receptor for advanced glycation end-products (RAGE). Diabetologia. 2010;53:2656-2666. https://doi.org/10.1007/s00125-010-1900-z.

84. Chen M, Curtis TM, Stitt AW. Advanced glycation end products and diabetic retinopathy. Curr Med Chem. 2013;20:3234-3240. https://doi.org/10.2174/09298673113209990025.

85. Zhou Z, Ju H, Sun M, et al. Serum Vascular Endothelial Growth Factor Levels Correlate with Severity of Retinopathy in Diabetic Patients: A Systematic Review and Meta-Analysis. Dis Markers. 2019;2019:9401628. https://doi.org/10.1155/2019/9401628.

86. Cheung N, Wong IY, Wong TY. Ocular anti-VEGF therapy for diabetic retinopathy: overview of clinical efficacy and evolving applications. Diabetes Care. 2014;37:900-905. https://doi.org/10.2337/dc131990.

87. Wirostko B, Wong TY, Simó R. Vascular endothelial growth factor and diabetic complications. Prog Retin Eye Res. 2008;27:608-621. https://doi.org/10.1016/j.preteyeres.2008.09.002.

88. Nagaoka T, Sato E, Takahashi A, et al. Impaired retinal circulation in patients with type 2 diabetes mellitus: retinal laser Doppler velocimetry study. Invest Ophthalmol Vis Sci. 2010;51:6729-6734. https://doi.org/10.1167/iovs.10-5364.

89. Burgansky-Eliash Z, Nelson DA, Bar-Tal OP, et al. Reduced retinal blood flow velocity in diabetic retinopathy, Retina. 2010;30:765-773. https://doi.org/10.1097/IAE.0b013e3181c596c6.

90. Arichika S, Uji A, Murakami T, et al. Retinal hemorheologic characterization of early-stage diabetic retinopathy using adaptive optics scanning laser ophthalmoscopy. Invest Ophthalmol Vis Sci. 2014;55:8513-22. https://doi.org/10.1167/iovs.14-15121.

91. Blum M, Eichhorn M, Vilser W. Haemodynamics and diabetic retinopathy. Klin Monbl Augenheilkd. 2005;222:463-70. https://doi.org/10.1055/s-2005-858422.

92. Curtis TM, Gardiner TA, Stitt AW. Microvascular lesions of diabetic retinopathy: clues towards understanding pathogenesis? Eye (Lond). 2009;23:496-508. https://doi.org/10.1038/eye.2009.108.

93. Khansari MM, Wanek J, Tan M, et al. Assessment of Conjunctival Microvascular Hemodynamics in Stages of Diabetic Microvasculopathy. Sci Rep. 2017;7:45916. https://doi.org/10.1038/srep45916.

94. Palochak CMA, Lee HE, Song J, et al. Retinal Blood Velocity and Flow in Early Diabetes and Diabetic Retinopathy Using Adaptive Optics Scanning Laser Ophthalmoscopy. J Clin Med. 2019;8:1165. https://doi.org/10.3390/jcm8081165.

95. Głowińska-Olszewska B, Urban M, Urban B, et al. The association of early atherosclerosis and retinopathy in adolescents with type 1 diabetes: preliminary report. Acta Diabetol. 2007;44:131-137. https://doi.org/10.1007/s00592-007-0253-7.

96. Klein R, Sharrett AR, Klein BE, et al. The association of atherosclerosis, vascular risk factors, and retinopathy in adults with diabetes : the atherosclerosis risk in communities study. Ophthalmology. 2002;109:1225-1234. https://doi.org/10.1016/s0161-6420(02)01074-6.

97. Peña AS, Liew G, Anderson J, et al. Early atherosclerosis is associated with retinal microvascular changes in adolescents with type 1 diabetes. Pediatr Diabetes. 2018;19:1467-1470. 
https://doi.org/10.1111/pedi.12764.

98. Zhou W, Yuan WF, Chen C, et al. Study on material base and action mechanism of compound Danshen dripping pills for treatment of atherosclerosis based on modularity analysis. $J$ Ethnopharmacol. 2016;193:36-44. https://doi.org/10.1016/j.jep.2016.07.014.

99. Iring A, Jin YJ, Albarrán-Juárez J, et al. Shear stress-induced endothelial adrenomedullin signaling regulates vascular tone and blood pressure. J Clin Invest. 2019;129:2775-2791. https://doi.org/10.1172/JCl123825.

100. Fleming I. Molecular mechanisms underlying the activation of eNOS. Pflugers Arch. 2010;459:793806. https://doi.org/10.1007/s00424-009-0767-7.

101. Busse R, Fleming I. Regulation of endothelium-derived vasoactive autacoid production by hemodynamic forces. Trends Pharmacol Sci. 2003;24:24-29. https://doi.org/10.1016/s01656147(02)00005-6.

102. Do DV, Wang X, Vedula SS, et al. Blood pressure control for diabetic retinopathy. Cochrane Database Syst Rev. 2015;1:CD006127. https://doi.org/10.1002/14651858.CD006127.pub2

103. Lim Y, Song TJ, Hwang W, et al. Synergistic Effects of Sanghuang $₫$ Danshen Bioactives on Arterial Stiffness in a Randomized Clinical Trial of Healthy Smokers: An Integrative Approach to in silico Network Analysis. Nutrients. 2019;11:108. https://doi.org/10.3390/nu11010108.

104. Han C, Jiang YH, Li W, et al. Study on the Antihypertensive Mechanism of Astragalus membranaceus and Salvia miltiorrhiza Based on Intestinal Flora-Host Metabolism. Evid Based Complement Alternat Med. 2019;5418796. https://doi.org/10.1155/2019.

105. Lam FF, Yeung JH, Chan KM, et al. Dihydrotanshinone, a lipophilic component of Salvia miltiorrhiza (danshen), relaxes rat coronary artery by inhibition of calcium channels. J Ethnopharmacol. 2008;119:318-321. https://doi.org/10.1016/j.jep.2008.07.011.

106. Antonetti DA, Barber AJ, Bronson SK, et al. Diabetic retinopathy: seeing beyond glucose-induced microvascular disease. Diabetes. 2006;55:2401-2411. https://doi.org/10.2337/db05-1635.

107. Nguyen TT, Shaw JE, Robinson C, et al. Diabetic retinopathy is related to both endotheliumdependent and -independent responses of skin microvascular flow. Diabetes Care. 2011;34:13891393. https://doi.org/10.2337/dc10-1985.

108. Horio N, Clermont AC, Abiko A, et al. Angiotensin AT(1) receptor antagonism normalizes retinal blood flow and acetylcholine-induced vasodilatation in normotensive diabetic rats. Diabetologia. 2004;47:113-123. https://doi.org/10.1007/s00125-003-1262-x.

109. Laspas P, Zhutdieva MB, Brochhausen $C$, et al. The M1 muscarinic acetylcholine receptor subtype is important for retinal neuron survival in aging mice. Sci Rep. 2019;9:5222. https://doi.org/10.1038/s41598-019-41425-5.

110. Sodhi P, Hartwick AT. Muscarinic acetylcholine receptor-mediated stimulation of retinal ganglion cell photoreceptors. Neuropharmacology. 2016;108:305-315. https://doi.org/10.1016/j.neuropharm.2016.04.001. 
111. lismaa TP, Kerr EA, Wilson JR, et al. Quantitative and functional characterization of muscarinic receptor subtypes in insulin-secreting cell lines and rat pancreatic islets. Diabetes. 2000;49:392-398. https://doi.org/10.2337/diabetes.49.3.392.

112. Duttaroy A, Zimliki CL, Gautam D, et al. Muscarinic stimulation of pancreatic insulin and glucagon release is abolished in $\mathrm{m} 3$ muscarinic acetylcholine receptor-deficient mice. Diabetes. 2004;53:17141720. https://doi.org/10.2337/diabetes.53.7.1714.

113. Kruse AC, Kobilka BK, Gautam D, et al. Muscarinic acetylcholine receptors: novel opportunities for drug development. Nat Rev Drug Discov. 2014;13:549-560. https://doi.org/10.1038/nrd4295.

114. Felder CC, Bymaster FP, Ward J, et al. Therapeutic opportunities for muscarinic receptors in the central nervous system. J Med Chem. 2000;43:4333-4353. https://doi.org/10.1021/jm990607u.

\section{Table}


Table 1

The potential effective ingredients of CDDP

\begin{tabular}{|c|c|c|}
\hline Compounds & Degree & Source \\
\hline Quercetin & 40 & Panax notoginseng \\
\hline Luteolin & 17 & Radix Salviae \\
\hline Tanshinone iia $^{a}$ & 6 & Radix Salviae \\
\hline Beta-sitosterol & 6 & Panax notoginseng \\
\hline Cryptotanshinone $^{a}$ & 5 & Radix Salviae \\
\hline Dehydrotanshinone II $\mathrm{A}^{\mathrm{a}}$ & 4 & Radix Salviae \\
\hline Dan-shexinkum d ${ }^{a}$ & 4 & Radix Salviae \\
\hline Dihydrotanshinlactone & 4 & Radix Salviae \\
\hline Tanshinone $\mathrm{i}^{\mathrm{a}}$ & 4 & Radix Salviae \\
\hline Stigmasterol & 4 & Panax notoginseng \\
\hline 2-isopropyl-8-methylphenanthrene-3,4-dione ${ }^{a}$ & 3 & Radix Salviae \\
\hline 4-methylenemiltirone ${ }^{a}$ & 3 & Radix Salviae \\
\hline $\begin{array}{l}\text { 2-(4-hydroxy-3-methoxyphenyl)-5-(3-hydroxypropyl)- } \\
\text { 7-methoxy-3-benzofurancarboxaldehyde }\end{array}$ & 3 & Radix Salviae \\
\hline Przewalskin b & 3 & Radix Salviae \\
\hline Isocryptotanshi-none ${ }^{a}$ & 3 & Radix Salviae \\
\hline Isotanshinone || ${ }^{\mathrm{a}}$ & 3 & Radix Salviae \\
\hline Miltionone $\mathrm{I}^{\mathrm{a}}$ & 3 & Radix Salviae \\
\hline Miltionone $\|^{\mathrm{a}}$ & 3 & Radix Salviae \\
\hline $\begin{array}{l}\text { (2R)-3-(3,4-dihydroxyphenyl)-2-[(Z)-3- } \\
\text { (3,4-dihydroxyphenyl)acryloyl]oxy-propionic acid }\end{array}$ & 3 & Radix Salviae \\
\hline Salvilenone I & 3 & Radix Salviae \\
\hline Tanshinone $\mathrm{Vl}^{\mathrm{a}}$ & 3 & Radix Salviae \\
\hline Poriferasterol & 2 & Radix Salviae \\
\hline Przewalskin a & 2 & Radix Salviae \\
\hline
\end{tabular}




\begin{tabular}{|c|c|c|}
\hline Compounds & Degree & Source \\
\hline Danshenol Ba & 2 & Radix Salviae \\
\hline Salvilenone & 2 & Radix Salviae \\
\hline Danshenspiroketallactone & 2 & Radix Salviae \\
\hline Deoxyneocryptotanshinone ${ }^{a}$ & 2 & Radix Salviae \\
\hline Miltipolone & 2 & Radix Salviae \\
\hline Miltirone ${ }^{a}$ & 2 & Radix Salviae \\
\hline Neocryptotanshinone ii ${ }^{a}$ & 2 & Radix Salviae \\
\hline Prolithospermic acid & 2 & Radix Salviae \\
\hline Ginsenoside rh2 & 2 & Panax notoginseng \\
\hline (+/-)-Isoborneol & 1 & Borneolum Syntheticum \\
\hline Poriferast-5-en-3beta-ol & 1 & Radix Salviae \\
\hline Sugiol & 1 & Radix Salviae \\
\hline Digallate & 1 & Radix Salviae \\
\hline $\begin{array}{l}\text { 5,6-dihydroxy-7-isopropyl-1,1-dimethyl-2,3- } \\
\text { dihydrophenanthren-4-one }\end{array}$ & 1 & Radix Salviae \\
\hline 3a-hydroxytanshinone II $\mathrm{a}^{\mathrm{a}}$ & 1 & Radix Salviae \\
\hline Formyltanshinone $^{1}$ & 1 & Radix Salviae \\
\hline 3-beta-Hydroxymethyllenetanshiquinone $\mathrm{a}^{\mathrm{a}}$ & 1 & Radix Salviae \\
\hline Methylenetanshinquinone ${ }^{a}$ & 1 & Radix Salviae \\
\hline Przewaquinone $c^{a}$ & 1 & Radix Salviae \\
\hline $\begin{array}{l}\text { (6S,7R)-6,7-dihydroxy-1,6-dimethyl-8,9-dihydro- } \\
\text { 7H-naphtho[8,7-g]benzofuran-10,11-dione }\end{array}$ & 1 & Radix Salviae \\
\hline Tanshinaldehyde ${ }^{a}$ & 1 & Radix Salviae \\
\hline Epidanshenspiroketallactone & 1 & Radix Salviae \\
\hline C09092 & 1 & Radix Salviae \\
\hline Neocryptotanshinone ${ }^{a}$ & 1 & Radix Salviae \\
\hline
\end{tabular}




\begin{tabular}{|lll|}
\hline Compounds & Degree & Source \\
\hline 1-methyl-8,9-dihydro-7H-naphtho[5,6- & 1 & Radix Salviae \\
g]benzofuran-6,10,11-trione ${ }^{\mathrm{a}}$ & & \\
\hline Salviolone & 1 & Radix Salviae \\
\hline $\begin{array}{l}\text { (6S)-6-hydroxy-1-methyl-6-methylol-8,9- } \\
\text { dihydro-7H-naphtho[8,7-g]benzofuran-10,11-quinone }{ }^{\mathrm{a}}\end{array}$ & 1 & Radix Salviae \\
\hline Tanshindiol B & 1 & Radix Salviae \\
\hline Przewaquinone Ea & 1 & Radix Salviae \\
\hline $\begin{array}{l}\text { (6S)-6-(hydroxymethyl)-1,6-dimethyl-8,9- } \\
\text { dihydro-7H-naphtho[8,7-g]benzofuran-10,11-dione }\end{array}$ & 1 & Radix Salviae \\
\hline DFV & 1 & Panax notoginseng \\
\hline
\end{tabular}

a Identified tanshinones.

\section{Figures}




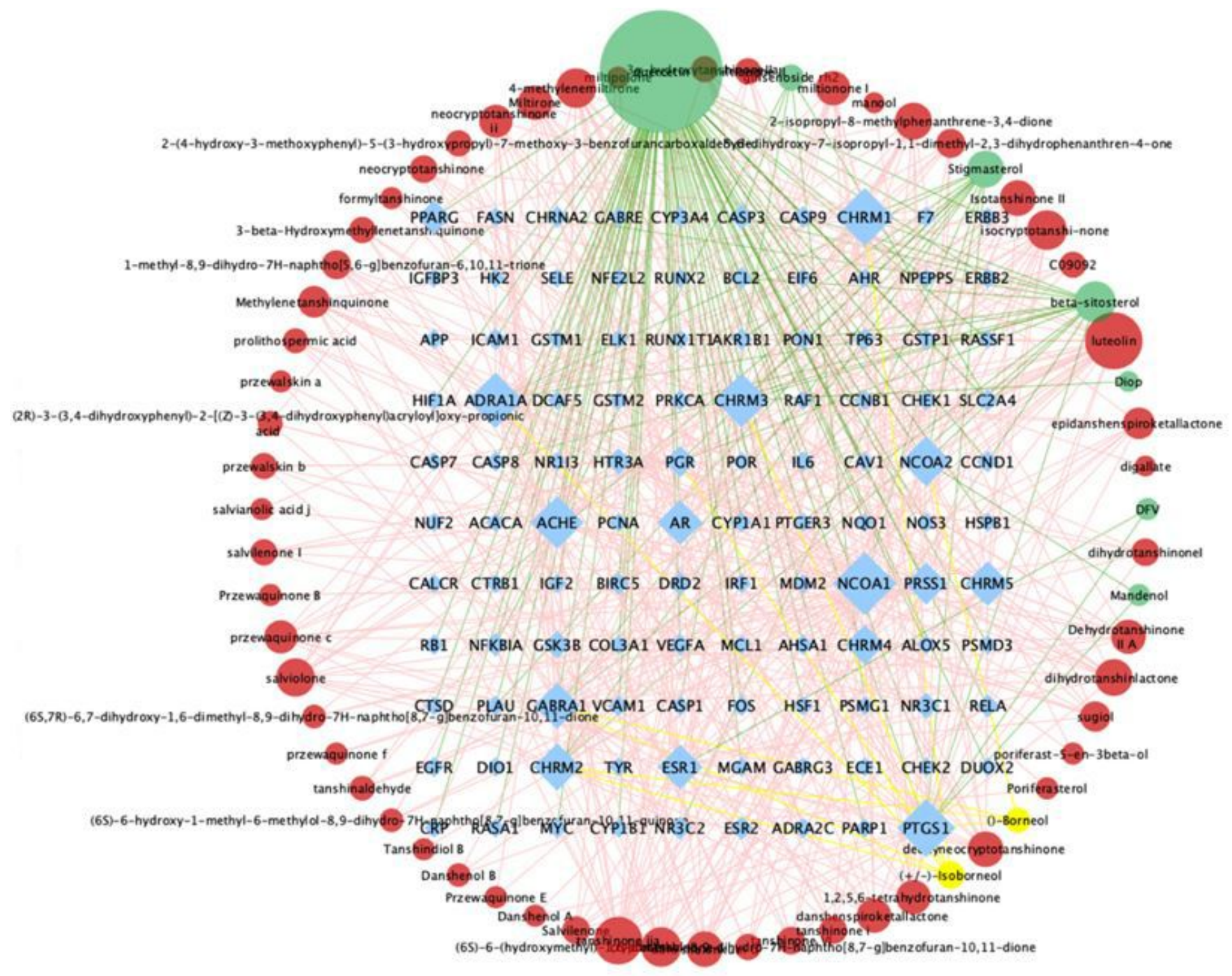

\section{Figure 1}

Compound-target network of CDDP: 64 compounds (the outer circle) and 109 targets (blue diamond) were identified. Fifty-five compounds were identified from RS (red dot), seven from PN (green dot), and two from BS (yellow dot). The size was based on the degree value. 


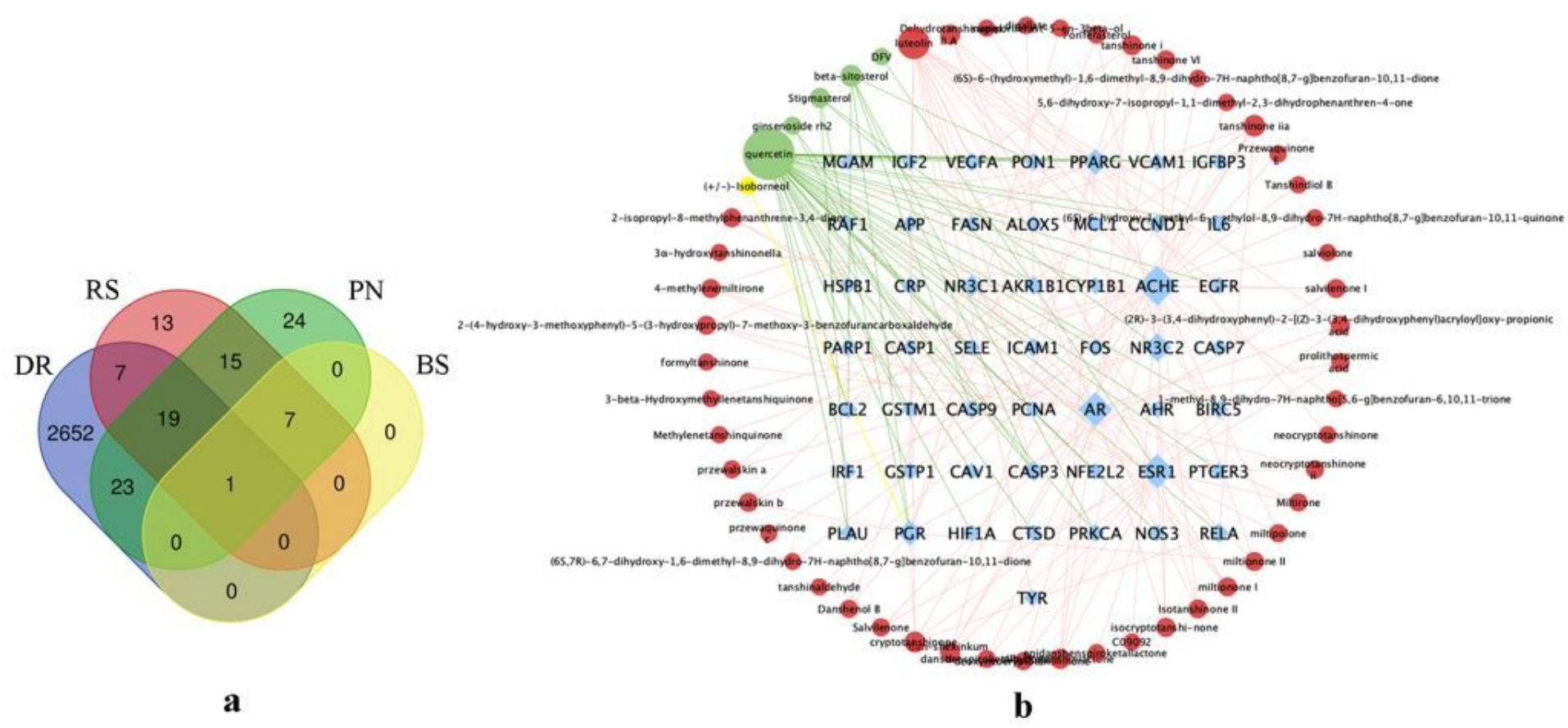

Figure 2

CDDP-DR common targets: (a) Venn map of CDDP-DR common targets; (b) Network of CDDP-compoundtarget-DR: Fifty common targets (blue diamond) were identified after merging the targets of CDDP and DR. Forty-eight compounds were identified from RS (red dot), five from PN (green dot) and one from BS (yellow dot). The size is based on the degree value.

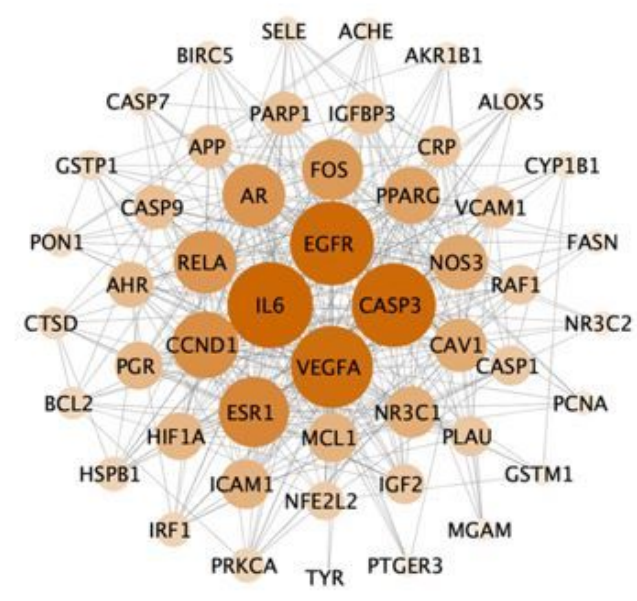

a

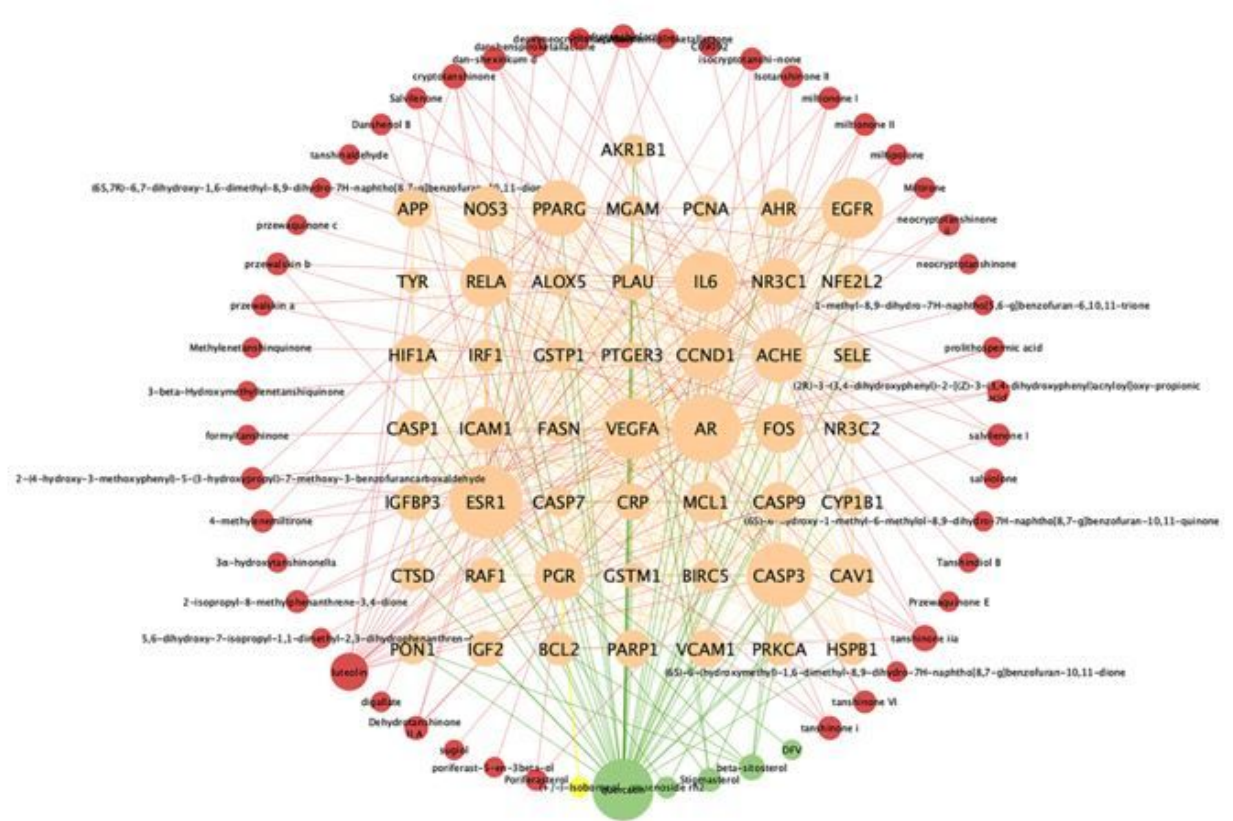

b

\section{Figure 3}


PPI network of CDDP against DR: (a) PPI network of the 50 candidates. The bigger and darker nodes represent higher degree value; (b) PPI merge network of CDDP against DR. PPI network was merged with the CDDP-compound-target-DR network (Figure 2b). The size is based on the degree value.

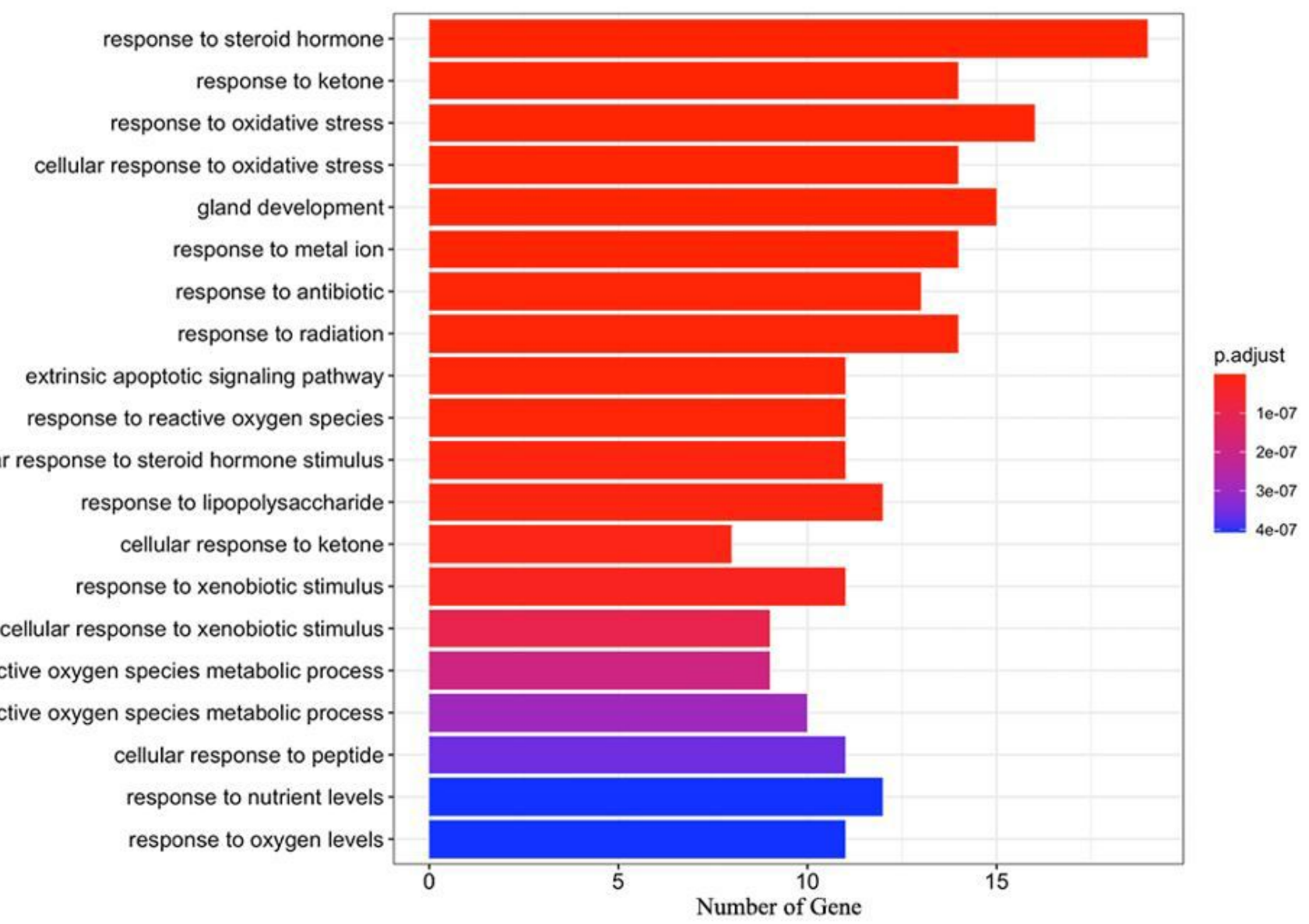

\section{Figure 4}

The biological process of candidate targets of CDDP against DR. The top $20 \mathrm{GO}$ functional categories with P-adjust $<0.05$ were selected. 


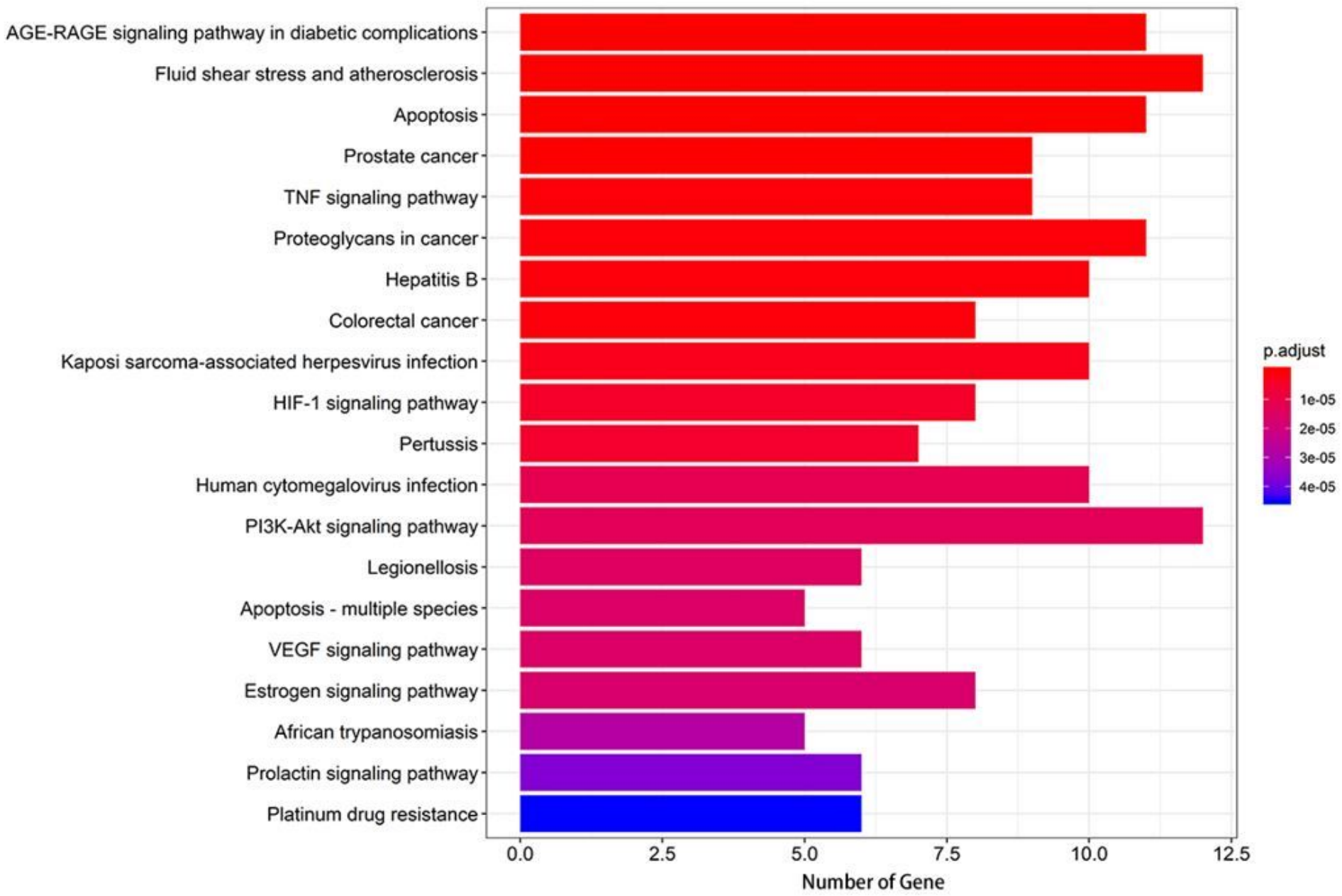

\section{Figure 5}

KEGG pathway enrichment of candidate targets of CDDP against DR. The top 20 pathways with P-adjust $<0.05$ were selected.

\section{Supplementary Files}

This is a list of supplementary files associated with this preprint. Click to download.

- Supplementary.pdf 